\title{
Téoros
}

Revue de recherche en tourisme

\section{L'Office des congrès et du tourisme du Grand Montréal} La plus ancienne organisation touristique du Canada

\section{Pierre Bellerose}

Volume 14, numéro 2, été 1995

Le tourisme : toute une histoire!

URI : https://id.erudit.org/iderudit/1075097ar

DOI : https://doi.org/10.7202/1075097ar

Aller au sommaire du numéro

Éditeur(s)

Université du Québec à Montréal

ISSN

0712-8657 (imprimé)

1923-2705 (numérique)

Découvrir la revue

Citer cet article

Bellerose, P. (1995). L’Office des congrès et du tourisme du Grand Montréal : la plus ancienne organisation touristique du Canada. Téoros, 14(2), 24-25.

https://doi.org/10.7202/1075097ar d'utilisation que vous pouvez consulter en ligne.

https://apropos.erudit.org/fr/usagers/politique-dutilisation/ 


\title{
L'Office des congrès et du tourisme du Grand Montréal La plus ancienne organisation touristique du Canada
}

\author{
Pierre Bellerose ${ }^{*}$
}

L'Office des congrès et du tourisme du Grand Montréal est né en octobre 1919, suite à l'initiative d'un groupe d'intervenants actifs dans l'industrie touristique montréalaise, qui s'était réuni dans le but de discuter de l'ouverture d'un bureau de tourisme et d'étudier les moyens à prendre afin de stimuler le tourisme automobile américain à destination de Montré̉al et de Québec. La promotion des réseaux routiers était assumée, dans le passé, par le ClubAutomobile, mais cetorganisme souhaitait maintenant qu'une autre organisation prenne la relève et élargisse le mandat.

Étaient présents, à cette première réunion, les représentants des entreprises et organismes suivants: Automobile Club of $\mathrm{Ca}$ nada (3 délégués), Fairwether's Ltd, British American Bank Note Co., Goodyear Tire \& Rubber Co., Northern Electric Company, Gilette Safery Rasor Company Ltd, Henry Birks \& Sons Ltd, Henry Morgan \& Co Ltd, Holt Renfrew Ltd, Mappin \& Webb Ltd, Canada Steamship Lines, Canadian National Railways, Hôtel Windsor, Hôtel Ritz-Carlton, Montreal Publicity Association, l'Association des commerçants, la Ville de Montréal, la Chambre de commerce, ainsi que le député E.M. Desaulniers, président de la Législature du Québec. Il s'agissait de la première rencontre au Canada dont l'objet principal était le tourisme.

Tous les membres présents étaient en faveur de la création d'un nouvel organisme de promotion touristique à l'échelle montréalaise et provinciale. Ce n'est que lors de la deuxième réunion, en décembre 1919, que la dénomination sociale *Tourists' Bureau of Montreal mo fut adoptée à l'unanimité. À la fin de cette même année, sept entreprises avaient déboursé chacune $500 \$$ afin de devenir membre de l'organisme, et d'autres s'étaient engagées

\footnotetext{
- Monsieur Pierre Bellerose est directeur de la recherche et du developpement aे l'Otfice des congres et du tourisme du Grand Montreal.
}

à joindre l'équipe, dont le Grand Trunk Railway, le Canadian Pacific Railway et l'Hôtel Queen's. En janvier 1920, la Banque de Toronto s'était engagée à acheter des espaces publicitaires dans toutes les publications du nouvel office.

Quelques mois après le début des opérations du Tourists' Bureau of Montreal, l'Adirondack Resort Association leur a proposé de s'associer à eux pour faire une campagne de publicité conjointe visant la promotion de leur destination.

Le bilan de la première année d'opération, présenté en novembre 1920 , fut très positif. Douze entreprises étaient devenues membres du Tourists' Bureau of Montreal (500 $\$$ chacune); un dépliant et une carte touristique de Montráll avaient été produits à 5000 exemplaires dont $90 \%$ avaient été distribués aux États-Unis et 1000 copies avaient été conservées pour être distribuées au congrès de l'Association des hôtels de New York dans les semaines suivantes.

En dernier lieu, la campagne de publicité conjointe avec l'Adirondack Resort Association avait été amorcée et tous les efforts promotionnels (surtout la publicité dans les journaux) avaient provoqué plus de 600 demandes d'informations touristiques en provenance de 26États américains, de trois provinces canadiennes et une demande provenait de l'Europe.

En avril 1924, l'organisme fut légalement incorporé sous la dénomination sociale de * Tourists' Bureau of Montreals, La ville devenant une destination de plus en plus populaire pour les congrès et les réunions, les administrateurs décidèrent de changer le nom, en janvier 1925 , pour *Montreal Tourist and Convention Bureau Incorporated\%. Les buts visés étaient les suivants: *Promouvoir le tourisme et les congrès dans la ville de Montréal et dans la province de Québec et, à cette fin, exploiter un ou plusieurs bureaux pour la diffusion des informations nécessaires, publier et mettre en circulation des cartes et documentations publicitaires de toutes sortes et accomplir tout acte et toute chose facilitant la promotion du tourisme....s

Dès le début de ses activités, l'Office de tourisme a orchestré des campagnes publicitaires dans de nombreux journaux américains. Les thèmes et les images vêhiculées étaient très variés, mais il est intéressant de constater à quel point l'hiver a été utilisé comme force d'attraction principale pendant pl usieurs années. Pendant la guerre, l'organisme a poursuivi ses activités promotionnelles malgrể le fait que la majorité des moyens de transports aient été réquisitionnés par les services de guerre et que les hôtels aient êté encombrés à cause de la crise du logement. Descampagnes de publicité furent lancées au Québec, au Canada et aux États-Unis et l'Office a su attirer des congrès dans la ville.

En 1944, le conseil municipal de Montréal a crée un département pour promouvoir le développement économique et touristique de la Ville. Lorsque Montréal obtint, en 1961 , la confirmation de la venue de l'Exposition universelle de 1967, l'administration municipale décida de mettre sur pied un Office municipal du tourisme.

Afin de ne pas dédoubler les efforts et d'éviter toute confusion, les deuxinstances conclurent une entente stipulant qu'à partir de cette date l'Office, qui portait à l'époque le nom d'Office des congrès et des visiteurs de Montréal, devait concentrer ses efforts sur la sollicitation et la coordination des congrès et des voyages de groupe, tandis que l'Office municipal serait essentiellement responsable de la promotion du tourisme d'agrément individuel et de l'accueil.

L'organisme a changé de dénomination sociale à deux reprises en 1963 et 1975 avant d'adopter, en 1982, le nom qu'il porte aujourd'hui.

En 1986, le nouveau président du conseil d'administration de l'OCTGM, monsieur Alain Cousineau a enclenchẻ un processus de planification stratégique qui a mis rapidement l'accent sur l'importance d'unifier 
les forces touristiques de Montréal pour assurer ainsi un plus grand leadership. C'est dans cet esprit et à la suite de cette démarche que l'OCTGM vivait une étape im= portante de son histoire en intégrant, en 1989 , le département de tourisme de la Ville de Montréal.

Parallètement à ces efforts, la Ville de Montréal et les deux paliers gouvernemen= taux inauguraient, en mai 1989 , le Centre INFOTOURISTE, un centre multifonctionnel d'accueil. Pour assurer la gestion de ce nouveau concept et toutes les fonctions d'accueil touristique de Montréal, la Ville de Montréal a créé, en 1988, un organisme para-municipal, la Société d'accueil et de renseignements touristiques deMontré̉al (SARTM) quiétait complètement indépendante de l'OCTGM. Mais, en janvier 1992, après une entente avec l'administration municipale, l'OCTGM a intégré toutes les fonctions d'accueil de la SARTM en créant une nouvelle direction de l'accueil en son sein.

Avec cette dernière étape a pris fin plus de trente ans de dédoublement des actions touristiques à Montréal et d'implication directe de la Ville de Montréal dans le champ de la promotion et de l'accueil touristique.

En 1994, l'OCTGM a organisé une soirée pour célébrer son $75^{\circ}$ anniversaire d'actvités et une exposition sur son histoire et celle du tourisme à Montréal qui a été inaugurée le 4 octobre 1994 au Centre d'histoire de Montréal.

$L^{\prime} O C T G M$ est entré dans sa $76^{\circ}$ année et, à l'orée de l'an 2000, avec un leadership consolidé, est plus que jamais prêt à assumer son rôle fondamental: faire la promotion du Montréal touristique et assurer l'accueil des touristes afin que cette région demeure une destination touristique de premier plan.

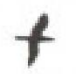

Le $50^{\circ}$ anniversaire de YOrganisation de Paviation civile intemationale: Marie-Janou Lusignan

Le 7 décembre 1994, le monde célébrait le $50^{\circ}$ anniversaire d'un des faits marquants de ce siècle: la naissance de l'Organisation de l'aviation civile internationale (OACI). L'OACI a bien érabli sa position dans le monde comme organe primordial pour l'amélioration de la sécurité, de la fiabilité et de l'efficacité des voyages aériens. Tout en étant un organisme technique, l'OACI a joué un rôle de pionnier parmi les institutions qui veillent sur la paix dans le monde. D'ailleurs, l'OACI est une institution spécialisée des Nations Unies. En vếrité, la création de l'OACI a contribué à l'instauration du système de l'ONU en 1945.

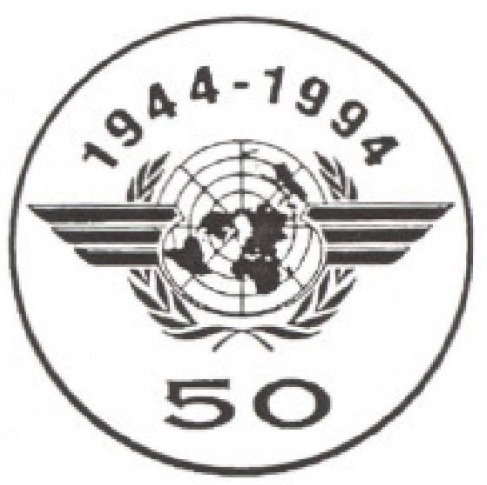

L'Organisation de l'aviation civile internationale est née à Chicago en 1944, où 52 nations se sont réunies afin de réaliser des ententes internationales. L'Organisation compte aujourd'hui 183 Etats contractants. Une bonne part de cette expansion est due au processus de décolonisation des années 1960 et 1970 , et plus récemment, au démembrement de l'Union soviétique en décembre 1991 après lequel 15 États exsoviétiques sont devenus membres de l'OACI. L'Organisation a son siège à Montréal et des bureaux régionaux à Bangkok, au Caire, ă Dakar, à Lima, à Mexico, à Nairobi et à Paris.

La charte qui lui a donné naissance, la Comvention relatrue à laviation àile internationale, est mieux connue sous le nom de Convention de Chicago. Initialement appelée OPACI(Organisation provisoire de l'aviation civile internationale), elle était fille de la Deuxième Guerre mondiale et petite-fille de la Première.

Le progrès énorme del'aviation pendant et entre les deux guerres, et en partie à cause de ces deux guerres, imposait l'adoption d'une réglementation internationale. Le monde avait besoin de l'OACI pour veiller à ce que les hostilités auxquelles avait contribué la technologie aéronautique ne dominent pas les airs après la fin de la guerre. Il fallait que l'ordre et la coopération viennent remplacer le conflit et la destruction.

L'OACI fournit le mécanisme de la coopération internationale dans le monde de l'aviation. Ses réalisations s'appliquent à des champs d'intervention multiples tels que l'établissement de normes, de pratiques et de procéduresinternationales dans les domaines techniques de l'aviation; la conception d'un système satellitaire destiné à répondre aux besoins futurs de l'aviation civile dans le secteur oommunications navigation, surveillance/gestion du trafic aérien (CNS/ATM); la planification régionale; la simplification des formalités et l'adoption de normes internationales de facilitation qui fixent le niveau maximum de formalités que les États peuvent exiger; l'amélioration des installations et des services d'aérogare a fin de faciliter et d'accélérer l'acheminement des passagers, des bagages et du fret aérien; la publication de statistiques, d'études et de manuels de formation; la coopération technique dans les pays en développement; l'unification des règles et l'élaboration de conventions de droit aérien international.

Ce texte est le resume d'un document transmis par le Bureau de l'infíormationdu public de l'Organisation de l'avation covile internationale 